Hospital Clínico Regional

de Concepción.

\title{
PNEUMOTORAX ESPONTANEO EN EL RECIEN NACIDO
}

Por el Dr. PROSPERo ARRIAGADA S.

Asesor Puericultor de la Maternidad,

y Di. ALBERTO AgUAYo A.

Médico Radiólogo Ayadante.

Se entiende por pneumotórax espontáneo a la existencia de aire en la cavidad pleural, sin que haya intervenido una causa externa para explicarla, sea ésta de orden médico - traumático. Con más propiedad hablando, no es una enfermedad, sino un sindrome que resulta de una perforación pieuropulmonar.

En cuanto a șu etiología, Pruvost distingue las siguiunttes variedades.

a) Tuberculose; b) Por infecciones a piógenos o anaerobios; c) Traumático (?), y d) Por lesiones quísticas o seudoquísticas pulmonares.

En relación a su extensión, sexá: a) Total, o b) Parcial. Este último, a su vez, ofrece varios tipos: apical, interlobar, mediastinal, basal, costomarginal. Se describe, además, una tercera variedad: c) Pneumotórax tabicados, que pueden ser areolares (por fibrina) o escalonados (como bolsillos superpuestos).

Desde el punto de vista del grado de colapso, hay pneumotórax completos e incompletos. Y en relación a la existencia o no de comunicación con los bronquios, se ven: a) pnetmotórax cerrados, y b) pneumotórax abiertos. Los pneumotóráx abiertos se subdividen en variedades que comunican ampliamente con los bronquios y otras en que existe una comunicación a válvula.

Castex y Mazzei exponen una clasificación clínico etiológica del pneumotórax espontáneo (refiriéndese al adulto). Describen los siguientes tipos: 
1) Pneumotórax espontáneo benigno por ruptura de burbujas pleurales, patrimonio de todas las alteraciones congénitas o adquiridas pulmonares que comprometen su elasticidad y su resistencia a la distensión. Con más propiedad debería llamarse Pneumotórax de los Cicatriciales.

2) Pneumotórax benigno recidivante, determinado por tuberculosis, enfisema o ampollas pleurales.

3) Pneumotórax del asma y enfisema. En el asma habría dos mecanismos: a) por procesos destructivos causantes o coincidentes con el asma (tuberculosis, infecciones bronquioalveolares, etc.), y b) por ruptura de enfisema vesicular crónico o agudo; o al llamado enfisema de erosión (vesículas subpleurales).

4) Hemopneumotórax espontáneo. Por ruptura de burbujas subpleurales, comprometiendo los vasos vecinos.

5) Formas dolorosas insólitas del pneumotórax y hemopneumotótax espontáneo. Sincopales o convulsivas; angiposas; braquiálgicas; sindtomes abdominales agudos; sindromes abdominales crónicos o lumbares.

6) Pneumotórax espontáneo en la tuberculosis. Son los más comunes y pueden verse en cualquier periodo de la enfermedad, aunque más frecuentes en las formas terciarias.

El sindrome clásico del pneismotórax espontáneo está constituído por los siguientes elementos:

A) Triada sintomatológica:

a) puntada de costado.

b) disnea intensa.

c) opresión toráxica.

B) Triada Signológica:

a) Abolición de las vibraciones vocales.

b) Hipersonoridad percutoria.

c) Ausencia de murmullo vesicular.

C) Triada radiológica:

a) Hiperclaridad intratoráxica.

b) Ausencia de trama pulmonar.

c) Línea límite que señala el borde del pulmón colapsado.

Cuando a la percusión hay hipersonoridad de sonido metálico y se suma a la auscultación soplo de timbre anfóri- 
co, es probable que el pneumotórax esté a presión y con comunicación a válvula.

Si radiológicamente se constata la existencia de un derrame con límite superior horizontal y que conserva su posición cualquiera la que sea la del enfermo, subiendo en la inspiración y con onda líquida al mover el paciente. se puede asegurar categóricamente la existencia de hidropneumotórax. Si 12. desviación del mediastino hacia el lado sano no guarta relación con la magnitud del derrame, a veces con formación de hernia mediastínica, debemos concluir que el pneumotórax está a preción; si esta diferencia se acentúa en exámenes sucesivcs, el pneumotórax ha de estar comunicado a válvula. En caso de dudas recurrir a las pruebas manométricas.

Desde el punto de vista radiológico, el pneumotórax enquistado del véttice puede confundirse con cavernas apicales. Sin embargo, una prolija investigación radiológica, clínica $y$ de laboratoric permiten formular el diagnóstico. Igualmente importante es la diferenciación radiológica del pneumotótax con el quiste aérco pulmonar congénito de tamaño gigante. Para ello hay que tener en cuenta los signos siguientes: En el quiste aéreo congénito no se aprecia el muñón pulmonar que sc aprecia en el pneumotórax por el colapso; en el quiste aéreo pulmonar, la imagen se ve terminar en la pared costal en forma de un ángulo obtuso y en el pneumotórax en forma de un ángulo agudo. En casos de dudas, el pneumotórax artificial permite seguir visualizando la imagen aérea del quiste junto al pulmón colapsado. Finalmente, la broncografía aporta un dato más de importancia en el diagnóstico diferencial.

Refiriéndonos en especial al pneumotórax espontáneo del recién nacido, objeto de esta publicación, hemos podido constatar dos casos en un período relativamente corto, al adoptar la norma de llevar a la pantaila radioscópica a todo recién nacido, cuya función respiratoria nos parecía perturbada.

Confirmanda la aseveración de los autores norteamericanos Salmon y Forbes, nos parece muy posible que la rareza del pneumotórax espontáneo del recién nacido, sea más bien aparente y que su poca definición clínica a tan temprana edad, cree confusiones con cuadros clínicos parecidos, escapándose la oportunidad de un estudio radioscópico y radiográfico, que habria rubricado con seguridad el cuadro. 
A1 buscar la causa de cualquier pneumotórax inexplicable, dos clases de factozes es necesario tener en consideración: 1) Un proceso patológico del árbol respiratorio, que debilite sus paredes, y 2) Una sobreventilación o distensión del alvéolo. En recién nacidos con deformidades congénitas del sistema respiratorio, infecciones pulmonares neonatales, etc., el procesc patológico es el más importante. En cambio, en casos con respiración difícil por secreciones del árbol tráqueobronquial (especialmente por mecanismo a válvula con enfisema distal), en atelectasias (con enfísema vicariante), en bemorragias intracraneanas, en enfermedades congénitas cardiacas, entra el factor de la sobredistensión.

Macklin ha demostrado experimentalmente en pulmones de gates y conejos el papel de la sobredistensión en la producción del pneumotórax. El aire pasa por múltiples y pequeñas rupturas del alvéolo hacia la vecindad de los vasos finos pulmonares: de allí siguen los vasos más y más gruesos hacia el mediastino. La ruptura de la pleura mediastínica hacia el espacio pleural puede ser una de las complicaciones. El aire puede pasar hacia el tejido intersticial del otro pulmón, hacia el cuello. al espacio retroperitoneal y de alli al peritonec o seguir los vasos inguinales determinando enfisema subcutáneo. En el recién nacido habría cuatro mecanismos posibles de producción de pneumotótax espontáneo: visceral.

a) Alvéolo - tejido intersticial - ruptura pleura

b) Alvéolo - tejido intersticial - mediastino ruptura pleura mediastinal.

c) Combinación de los dos mecanismos anteriores.

d) Existencia de un proceso patológico fulmonar local que determine el pneumotórax a tensión.

Los síntomas y signos del pneumetórax espontáneo del recién nacido, no exigen mayores comentarios, aunque vale 1a pena recordar que la disnea y cianosis son intensificadas apreciablemente por la compresión mecánica del aire mediastinal sobre los grandes vasos. En los casos de sobreventilación y distensión, el enfisema mediastinal es más probable que preceda al pneumotórax y la causa de esa sobreventilación o distensión es la que determiná la dificultad respiratoria y no el pneumotórax, que es más bien su consecuencia.

El diagnóstico radiológico del pneumotórax es bastante fácil, como asimismo la existencia de enfisema mediastinal. 
Su tratamiento es exclusivamente conservador; usando mezclas de anhidrido carbónica y oxígeno, oxígeno puro y estimulantes cardiovasculares. graves.

La aspiración del aire se reservà para los casos más

En la revisión de la literatura nacional que hemos efectuado, no hemos encontrado referencias a pneumotórax espontäneos en recién nacidos, hecho que atribuimos a la confusión de este cuadro patológico con otros, propios de esta edad, que por su gran frecuencia dominan la morbilidad y causa de mortalidad del recién nacido Nos referimos a los traumatismos obstétricos cráneoencefálicos, asfixias neonato$\mathbf{r u m}_{2}$ bronconeumonias, cardiopatías congénitas, atelectasias pulmonares etc. Los solos síntomas y signos clínicos, en su gran mayoría, no hacen posible al diagnóstico, y es entonces cuando el estudio radiológico es de inestimable valor en el diagnóstico diferencial.

Exponemos a continuación la observación clínica, con sus documentos radiológicos y de necrepsia. de dos casos estudiados en la Maternidad del Hospital Clínico Regional de Concepción.

CASO N. 1. - M. T. C. Obs. N. $936816-44$. Nace el 19 de noviembre de 1944 a las 12 horas en la Maternídad del Hospital Clínico, con asfixia azal inteasa, que se logra vencer (?) más o menos a los 15 minutos con estímulos cutáneos, estimulantes cardiacos $y$ respiratorios y respiración artificial. Peso: 2,950 grs. Flacenta: 490 gts. La madre de 33 años (F. C. Obs. N.9 36819-44), sana; serología negativa; radioscopía de tótax normal, en nopiambre de 1944; dos hijos muertos: coqueluche, trástomo nutritivo agado; seis hijos sunos. El padre de 44 años, sano. betedor, sin serología. No hay consanguinidad ni insania. El 20 el recién nacido está fícío, cianótico, hír potónico, fontanela depresible, 88 respiraciones tegulates, 108 contracciones cardiacais, hígado a un través de dedo, estertores fízos inspiratorios en la base izquierda. Radioscopía de Tórax: en la zona paravertebral de la mitad superior izquierda se aprecia zona de menor transpatencial, que impresiona como atelectasia; testo es normal. La radiografia del tórax (N.9 36560) evidencia pueumotornx izquierdo que abarca del véstice a la base. Se deja con oxigeno. a permanencia, cotamina, vitamina $\mathrm{K}$, calentamiento, leche materna extraída $\mathrm{y}$ fraceionada. El 21 bzly quejido, polipnea, wómitos verdosos, rechraza el a!imento. cianosis mercada. estertores finos base izquierda, tonos cardíacos claros, distensión abdominal con diseñamiento de asas, timpanismo: temperatara rectal en 1 a mańana $35.5^{\circ}$, en la taxde $35.8^{\circ}$. Surfatiazol $0.10 \mathrm{gr}$. por kgr. de prso, coramina $y$ cafeína alternados, oxigeno a permanencia, lecthe materna 


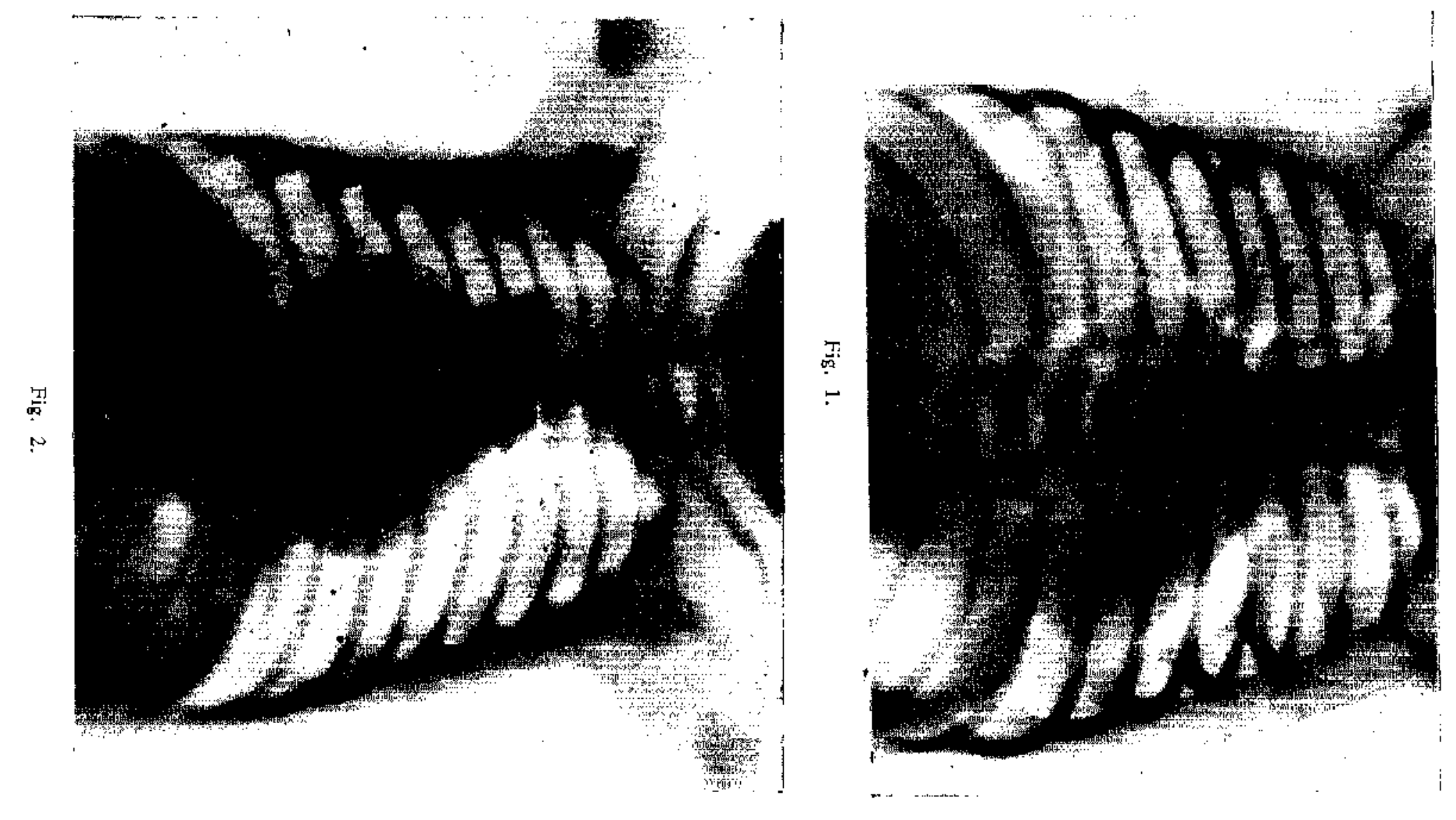


F liqnidos por cuchacaditas o gotario. El 22 la temperatura se eleva a 38.2 , las condiciones generales se agravan; 180 contracciones caltiacas, pulmones sin cambios, el hígado crece a dos traveses, aparee circulación vienosa colateral. vertical, tóracoabdominit, cianosis. La radiografía de tórax $\mathrm{N}^{9} 36721$ (figura $N \cdot 9$ 1) informa que en ambos paténquimas. especialmente mitades soperiores, bay opacidad; se aprecia en las bases. regiones laterales, usencia de parénquima, por pneumotórax bilateral. Faliece el dia 23 de noviembre de 1944.

Protocolo de Antopsia N.9 517 (Insrituto de Anatomía Patológica de 1a Universidad de Concepción), Diagnóstico Anátomopatológico: Bronconenmonía. Enfisema agudo. Pneumotórax bilateral.

Descripción exrerior: Recién nacido de sexo femenino, sin malformacicnes externas. Cortón ambilical ligado, de 5 cms., en momificación.

Cráneo: Suturas y fontanelas ampliamente abiertas. Hoz del cerebro y tienda del cerẹbelo intactas. Cenebro, ecrebelo y bulbo nada de especial.

Organos del cuello: Sin malformaciones.

Tórax: Se zutopsió bajo el agua. Al abrir la cavidad toráxica silen abandantes burbujas de aise, penetrando agua en las cavidides pleutales. Los. palmones se encuentran algo rettaidos hacia el bilio, especialmente el izquierdo. En ias cavidades no hay liquido ni atherencias. Pulmones de tamaño corriente, consisteacis firme, especialmente el izquierdo. $L_{a}$ superficie es de cotor rojo oscuro, salpicada en parte de zonas rasadas $y$ que hacen relieve. En la carà interra y borde anterior de ambos pulmones, especialmente el detecho, se eprecian numerosas bulas de diferentes tamaños.

También se encuentran estas bulas en todo el mediastino. especialmente en su parte anterior. Conazón pequeño, sin malformiciones, Abdomen. Cavidad sin liquido ni adherencias. Higado de tamaño y consistencia cotriente, snperficie lisa, brillante, café amarillento. Al corte, dibajo conservado.

Bazo pequetio con la superficie lis:l y brillante, tojo oscura. Al corte, djbajo bien visible. Estómago, intestino, mesenterio, páncreas, saprarrenales y riñones nada de especial.

Extremidades: Linca de osificación de ambos fémures recta, delgada y tosada.

Exaren histolögico pulmonar: Fulmón izquierdo: intersà congestión. Numerosas hemorragias dentro de los alvéolos.

Palmón derecho: Cuadzo en pzrte parecido, pero llama la atención partes enfisematosas $y$ aisladas partes neurnónicis.

CASO N. 2. - E. C. D. Obs. N: 35080-44. Nace el 12 de actubre de 1944 en la Maternidad de? Hospital Clínico de Concepión, por versión interna a causa de falta de encajamiento. Pesó 2.140 grs, Y Iät placenta 550 grs. (previa lateral anatómica). No bubo manifestaciones de sufrimiento fetal. La madre (G. D. Obs. N.9 35077-444), de 26 atuos de edad. con sto 
rologia negativa y rádioscopía de tórax normal; un aborto en 1943. El padre de 23 aîtos. sano, serología negativa en 1943. El abuelo matemo axusa subirculosis pulmonar. No hay consanguinidad de los padres ni dacos de inarrí familist. El 13, el recién nacide to ofrece ontas particularidades que tendencia al enfriatriento; se indica calztadotes, vitamina $K$. leche humana 5 grs. cada dos horss, $\mathrm{E}_{\leftarrow} 14$ iparece polipnea quejumbrosa, zianosis de labios" y extramidides, submatidez de la base pulmonar derecha, lluvia de estertores. finos de la ritad inferior del mismo lado y broncofonia diz todo ese campo. apagamiento respiratorio : izquirtda. A !a cadioscopia de tórax se encuentrs e! poeumotórax izquierdo con rechazo del mediastino a la derecha. La tadiografis N.P 34496 (Fig. N." 2) canfi:ma la radioncopía y permite, además, zpreciar discteta ojacidad de todo e! hemitćrax derecho. Si agrega a la terapéutica tónicos cardíacos $y$ oxígeno a parmanencia. Fallece al 15 , sin evidencír cambies en sus condiciones ginerales. Evolución absolutamente afebriy.

Protocolo de Antopsia N.o 449 (Instituro de Anatomía Patológica de La Univarsidad de Concepción), Díagnóstico Anátomopatológico: Prematuridá, tronconeumonía, Pneumotórax izquicrdo.

Descripción exterior: E'rematuro de sexo femenino, sin malformaciones externas. Cordón arrbilical de 5 cms., ligado en buenas condiciones, algo nromifirado.

Cráneo: Suturas y fontanelas ampliamente abiertas. Hoz del zerebro y tienda del cerebelo intictas. Cerebro, cerebelo y bulbo nada de especial.

Organos del cuello: Sin malformaciones.

Tótax: Cavidades sin liquido ni adherencias. Al abrir la cavidad toráxica se apteciz que el palmón izquierdo está algo retraido bacià el hilio. Los. pulmones son de tamaño corriente, de consistencia uniformemente aumentada, al derecho y el izquierdo sólo a nivel del lóbulo inferíor. En el lóbłulo strparior izquierdo se aprecia, principalmente a nivel de su borde anterior y cara intem 2. pequeñas vesicalas intersticiales, siendo algunas del tamaño da un garbanzo $y$ el resto no mayores de un grano de mijo. Al corte se afrecia el parénquima de ambos pulmones uniformemente de color rojo. A la expresión de todos los lóbalos del puimón derecho y del lóbulo inferior izqujerdo sale abundante líquido sanguinolento acompañado de algunas gotitas de pus. Corizón de tamaño corraspondiente a la edad del cadáver, de consistencia firme. sir malformaciones.

Abdomen: Cavidades sin liquido ni adberencias; higado de tamaño $\infty^{-}$ rrespondiente a la edad del feto, superficie lisa y brillante de color rojizo, al corte dibajo consérvado. Bazo pequeño, de superficie lisa y brillante, de color rojo; al corte el dibujo es bien visibie. Estómago, indestino, medenterio, pán. creas y suprarrenales nata de especial. Riñones lobulados, con infartos úricos; en la cara anterior del riñón izquierdo se encuentran spis a siete vesiculitas del tamaño de un grano de pimienta, conteniendo un liquido claro. Orga- 
nojs polvianos sin m lformaciones. Extremidades: líneas de osificación dz ambers fémures deigindas. rectas y rosadas

Examen bistológico pulmonar: Focos bronconeumónicos y bronqueolitis purulenta 2 el löbujo inferior derecho e izquierdo: en parte atelectasias. En el lóbulo superior izquietdo. cerca del enfisema, se ven a'lgnas partes con condnetos aiveolares muy dilatados (enfisematosos) y en la vecindad atelectasia; pero no $\$ z$ ye un marcado proceso inflimatorio ni tampoco mayor secración de los bronquios.

\section{RESUMEN}

Los autores presentan dos casos de pneumotórax espontáneo en recién nacidos. Ambos niños fallecen a los pocos días del nacimiento $\left(3 .^{\circ}\right.$ y $4 .^{\circ}$ por bronconeumonía). EI diagnóstico del preumotórax se hizo radiclógicamente y fué comprobado en la necropsia. Hacen una revisión de la etiología, patogenia y cuadro clínico del pneumotórax espontáneo, con especial referencia a su producción en niños recién nacidos.

Sugieren, mientras obtienen más experiencia al respecto, las siguientes conclusiones:

1. ${ }^{9}$ Tener presente la existencia del sindrome en todo recién nacido con trastornos de su función respiratoria.

$2 .^{\circ}$ No confiarse exclusivamente de los datos obtenidos por la clinica para fundamentar el diagnóstico de pneumotórax espontáneo del recién nacido, pues a esta temprana edad hay múltiples cuadros que afectan la función respiratoría y que son difícilmente dilucidables sin el empieo de otros recursos.

3. Llevar a la pantalla radioscópica a tcdos los niños recién nacidos, con disfunción respiratoria. La radiografía es un elemento de indudable valor al precisar más los datos aportados por la radioscopia.

4. Piensan que dicho esquema de trabajo pondrá err evidencia muchos casos de pneumotórax, que actualmente pasan desapercibidos.

\section{BIBLIOGRAFIA}

CASTEX y MAZZEI. - Nuevos estudios sobre los Pneumotórax *pontáneos. 1937.

RepetTI. PABlo. - Día Médico. Año XVI. N. 23, pág. 579.

NEIRA, MANUEL. — Areh. Hospital R, del Ría." Tomo II. N.*2, pág. 115. SAlMON-FORBES: - The Journat of Pediatrics. Vol, 23, N. 1, pafg. 50. PRUVOST, PIERRE. - Encyclopédie Médico-Chirurgicale. 